Research Paper

\title{
Identification of Tmem I 0 as a Novel Late-stage Oligodendrocytes Marker for Detecting Hypomyelination
}

Wanxiang Jiang1\#, Wanchun Yang1\#, Weiwei Yang1, Junyan Zhang1, Dejiang Pang1, Lingxue Gan1, Liping Luo $^{1}$, Yingjun Fan², Yanhui Liu ${ }^{2 \bowtie}$, Mina Chen ${ }^{1 凶}$

1. State Key Laboratory of Biotherapy, West China Hospital, Sichuan University, Chengdu 610041, China.

2. Department of neurosurgery, West China Hospital, Sichuan University, Chengdu 610041, China.

\# These authors contributed equally to this paper

$\square$ Corresponding author: Yanhui Liu, PhD, Professor, Department of neurosurgery, West China Hospital, Sichuan University, Chengdu 610041, China. Email: liuyanhui9@gmail.com; Mina Chen, PhD, Associate Professor, State Key Laboratory of Biotherapy, West China Hospital, Sichuan University, Chengdu 610041, China. Tel: +86-28-85164094 Fax: +86-28-85164095, Email: chenmina2010@gmail.com

(c) Ivyspring International Publisher. This is an open-access article distributed under the terms of the Creative Commons License (http://creativecommons.org/ licenses/by-nc-nd/3.0/). Reproduction is permitted for personal, noncommercial use, provided that the article is in whole, unmodified, and properly cited.

Received: 2013.08.29; Accepted: 2013.11.20; Published: 2013.12.10

\begin{abstract}
Oligodendrocytes ensheath axons to form compact insulating multilamellar structures known as myelin. Tmem IO is a novel type I transmembrane glycoprotein that is highly expressed in oligodendrocytes and whose biological function remains largely unknown. Furthermore, the expression pattern of Tmem 10 remains a matter of some controversy. Given the inconsistency of its expression pattern and the lack of validated specific antibodies, Tmem I0 is not widely accepted as a marker for mature oligodendrocytes. As a means to solve these problems and to aid future studies of oligodendrocyte-associated diseases, we have generated a highly specific Tmem IO antibody. Using this Tmem 10 antibody, we clarify that Tmem 10 protein is firstly expressed at 2 weeks in the postnatal mouse brain with age-related increase, only in the central nervous system (CNS). We also reveal that Tmem 10 is expressed specifically in late stage oligodendrocytes and later than MAG, a late-stage myelin marker. Finally, we show that Tmem 10 co-expresses with MOG- and MBP-positive myelin fibers and is dramatically reduced in a hypomyelination mouse model. In conclusion, our study demonstrates that Tmem 10 can be used as a specific marker for myelinating oligodendrocytes and perhaps for the evaluation of myelination diseases, such as multiple sclerosis.
\end{abstract}

Key words: Tmem10; polyclonal antibody; late stage oligodendrocytes maker; Myelination

\section{Introduction}

Oligodendrocytes are glial cells that are best known for their role in axonal ensheathment and myelin assembly within the mammalian central nervous system. When viewed in cross-section, myelin sheaths appear as multilamellar stacks of spirally wrapped cell membranes. The compacted structure of these sheaths provides myelinated axons with a high membrane resistance and a low capacitance, which are required for the rapid conduction of saltatory impulses [1]. In addition, a growing body of work has demonstrated that oligodendrocytes are also vitally important for maintaining axonal integrity, a function that is independent of myelination [2-4]. Furthermore, the dysfunction of oligodendrocytes is associated with many neurological diseases, such as multiple sclerosis $[5,6]$ and leukodystrophy [7]. However, the precise mechanisms by which oligodendrocytes ensheath axons during myelination and maintain axonal integ- 
rity are not fully understood. The identification of oligodendrocytes specific proteins (markers), particularly those are expressed in myelinating oligodendrocytes, would help to reveal the process of myelination. One such candidate gene is Tmem10, also known as Opalin.

By gene profiling, Tmem10/Opalin was originally identified as a gene whose expression is enriched in oligodendrocytes of the CNS, but not expressed in the peripheral nervous system [8]. But its biological function remains unclear. Tmem10 gene encodes a novel type I transmembrane protein and its protein sequences in mouse, rat and humans are conserved [8], suggesting that it might play a critical role in these species. It has been suggested that Tmem10 is selectively expressed in mature oligodendrocytes and touted as a new marker for myelinating oligodendrocytes. However, it is also reported that Tmem10 is expressed in immature oligodendrocytes $\left(\mathrm{O}^{+}\right)$[8-10]. Given the inconsistency of the results from different laboratories and the lack of validated specific antibodies, Tmem10 is not widely accepted as a marker for mature oligodendrocytes. As a first step towards the understanding of the biological function of Tmem10, we generated and validated the specificity of Tmem10 antibody. We show that Tmem10 is expressed later than other myelin proteins, such as MBP, MOG and MAG, and primarily expressed in mature oligodendrocytes. In addition, we show that Tmem 10 protein is dramatically reduced in a mouse model with hypomyelination, suggesting that Tmem10 is a useful marker for assessing myelination.

\section{Materials and methods}

\section{Reagents}

Anti-MBP and anti-myc antibodies were purchased from Calbiochem (San Diego, CA, USA); anti-Beta-Catenin antibody from Sigma-Aldrich (St. Louis, MO, USA); anti-HMGCS2 antibody from LifeSpan BioSciences (Seattle, WA, USA); anti-MOG, anti-MAG, anti-Olig2, anti-NG2, anti-GAPDH and anti-beta actin antibodies from Millipore (Billerica, MA, USA). IPTG (isopropyl $\beta$-D-thiogalactoside) was purchased from Merck (Darmstadt, Germany). Polyethylene glycol (PEG 8000) and Freund's complete adjuvant and Freund's incomplete adjuvant were from Sigma-Aldrich (St. Louis, MO, USA). Dulbecco's Modified Eagle Medium (DMEM), fetal bovine serum (FBS) and Neurobasal Media were from Gibco (Invitrogen, Carlsbad, CA, USA). Lipofectamine ${ }^{\mathrm{TM}} 2000$ in vitro transfection reagent was from Invitrogen (Carlsbad, CA, USA). Anti-mouse and anti-rabbit IgG antibodies conjugated to horseradish peroxidase were from Pierce (Rockford, IL, USA). Protease Inhibitor
Cocktail was from Calbiochem.

\section{Cloning of Tmem I 0}

Total RNA was isolated from 4-week-old mouse cortices using Trizol reagent (Invitrogen). Reverse transcription reactions were carried out with reverse transcriptase according to the manufacturer's instructions (Fermentas). The full-length Tmem 10 cDNA was subcloned by amplifying a full-length cDNA library with an appropriate primer pair (Tmem10-F-5'-CCGGTCGACGATGAGTTTTTCACT GAACT-3' and Tmem10-R-5'-TCAGCGGCCGCTG ATGCGGTGACATCATTCT-3') and inserting the amplified product into the mammalian expression vector $\mathrm{pRK} 5$-myc. The DNA sequence encoding the C-terminal fragment of Tmem10 was amplified using the pRK5-myc-Tmem10 vector as template and the following PCR primers: Tmem10-C-F-5'-ACTGTC GACatgGAGGAGACTGAGATACC-3' and Tmem10C-R-5'-TATGCGGCCGCTTCTAGGCTCAGGCTGG GT-3'; the PCR product of the expected size was cloned into the PET22b-His vector to generate the PET22b-Tmem10-His recombinant plasmid.

\section{Expression and purification of recombinant Tmem I 0-His protein}

The recombinant plasmid PET22b-Tmem10-His was transformed into Escherichia coli BL21 cells. The transformed bacteria were induced with $0.25 \mathrm{mM}$ isopropyl $\beta$-D-thiogalactoside (IPTG) at $35{ }^{\circ} \mathrm{C}$ for 3 hours. All samples were collected in PBS with $1 \%$ Triton X-100 and proteinase inhibitors and analyzed by Coomassie blue staining following $12 \%$ SDS-polyacrylamide gel electrophoresis. After induction of the Tmem10-His fusion protein, the recombinant protein was purified. Briefly, BL21 cells transformed with PET22b-Tmem10-His were cultured in $500 \mathrm{ml}$ Luria-Bertani (LB) medium at $35^{\circ} \mathrm{C}$. IPTG was added to a final concentration of $0.25 \mathrm{mM}$, and the cells were incubated for 3 hours. Following induction, the cells were collected and sonicated to create a cellular extract. The cellular extract was added to a pre-equilibrated column and washed with buffer $(60$ $\mathrm{mM}$ imidazole, $0.5 \mathrm{M} \mathrm{NaCl}$ and $20 \mathrm{mM}$ Tris- $\mathrm{HCl}, \mathrm{pH}$ $8)$. Then the recombinant protein was eluted from the column with elution buffer $(1 \mathrm{M}$ imidazole, $0.5 \mathrm{M}$ $\mathrm{NaCl}$ and $20 \mathrm{mM}$ Tris-HCl, $\mathrm{pH}$ 8)[11].

\section{Generation and purification of a Tmem 10 polyclonal antibody}

Anti-mouse Tmem10 antibody was generated in female New Zealand White rabbits following three subcutaneous injections of the purified Tmem10-His fusion protein as the antigen. Briefly, $200 \mathrm{mg}$ Tmem10-His fusion protein was emulsified in an 
equal mass of complete Freund's adjuvant (Sigma-Aldrich) and subcutaneously injected into a New Zealand White rabbit; this process was repeated twice at two week's interval in the same rabbit. The immunized rabbit was bled from its carotid arteries one week after the last immunization, and antiserum was obtained by centrifuging the blood at $1,000 \mathrm{~g}$ for 10 min. To purify the Tmem10 antibody, we first generated a GST-Tmem10 fusion protein, which was then used to purify the anti-Tmem10 serum using the AminoLink Plus Immobilization Kit (Thermo).

\section{Animals}

Experimental mice (from a mixed C57/6J and 129 backgrounds) and rabbits (New Zealand White) were bred and maintained under standard housing conditions in the animal facility of the West China Hospital of Sichuan University. All mouse work was carried out in strict accordance with the Animal Care and Use Committee guidelines of Sichuan University West-China Hospital, and the protocol was approved by the Animal Care and Use Committee of Sichuan University West-China Hospital. As previously described, Rheb1 Nestin-Cre knockout mice were generated in our lab [12]. Mice carrying a "floxed" Rheb1 were crossed with Nestin-Cre transgenic mice to create a CNS-specific deletion of Rheb1 [12]. Tmem10 knockout mice were generated with a knockin/knockout strategy by inserting a Cre gene into the locus right after the Tmem10 promoter in our lab (Wanxiang Jiang and Weiwei Yang, unpublished).

\section{Cell cultures}

BL21 and XL-blue cells were kindly provided by Johns Hopkins University. HEK293 cells were purchased from ATCC (USA) and maintained in MEM with $10 \%$ FBS. Transfections were performed using lipofectamine $^{\mathrm{TM}} 2000$ when cells were $80-90 \%$ confluent. Cell extracts were harvested 72 hours after transfection by treating cells with lysis buffer (PBS with $1 \%$ Triton and proteinase inhibitors).

OPCs, astrocytes and neurons were cultured according to standard protocols. Primary cultures of rat oligodendrocytes were prepared as previously described [13]. Briefly, cerebral cortices from postnatal day 2 rats were dissected out, minced, digested for 15 min at $37{ }^{\circ} \mathrm{C}$ in S-MEM (Gibco, Grand Island, NY) containing $0.1 \%$ Trypsin (Sigma) and plated in tissue-culture flasks coated with $100 \mathrm{~g} / \mathrm{ml}$ poly-L-lysine (Sigma). Cell cultures were maintained in DMEM containing $10 \%$ fetal bovine serum at $37{ }^{\circ} \mathrm{C}$ under humidified air with $5 \% \mathrm{CO}_{2}$ for about 10 days, with a medium change every 3 days. On the tenth day, the cell culture flasks were sealed and shaken. The media containing the detached cells were collected and plated on fresh tissue-culture dishes. The next morning, the culture medium was changed to defined media consisting of B27 (Invitrogen/Gibco \#17504-044)/NBM(Invitrogen,\#21103049), each containing $10 \mathrm{ng} / \mathrm{ml}$ PDGF AA (Pepro Tech 100-13A) and maintained until confluent. And then split into $60 \mathrm{~mm}$ dishes, when OPCs are about $30-40 \%$ confluent, PDGF AA was withdrawn and the OPCs began to differentiate, and we harvested the cells at indicated time points (Day) after PDGF AA withdraw for western blotting or immunostaining.

For the astrocytes cultures, cerebral cortices from postnatal day 2 rats were dissected in HBSS buffer, digested in $0.25 \%$ Trypsin and plated on gelatin-coated plates. Unattached cells were knocked off each time the media was changed with modified DMEM/F12 culture medium with 10\% FBS, 1\% glutamine, and gentamicin antibiotic. The plates were incubated at $37^{\circ} \mathrm{C}$ in $5 \% \mathrm{CO}_{2}$ for 2-3 days without disruption and the medium was changed in each flask every 2-3 days until confluency was achieved (after approximately 6-7 days). The cells were harvested for Western blotting.

Neuron cultures were prepared from the cortices of embryonic day 16 mice and plated in 6-well culture plates coated with poly-L-lysine. After 2 days in culture, $10 \mathrm{mM}$ cytosine arabinoside was added for 24 hours to prevent glial proliferation. The neurons were subsequently maintained in serum-free Neurobasal medium (Gibco) containing glutamax, horse serum and B27.

\section{Western blotting analysis}

Western blotting was performed according to standard procedures. To extract protein from cultured cells or tissues isolated from distinct regions of the mouse brain at specific ages, samples were sonicated in lysis buffer (either PBS with 1\% Triton X-100 and proteinase inhibitors for culture cells or $2 \%$ SDS with proteinase inhibitors for tissues). The protein concentration of each extract was measured using the BCA Protein Assay kit (Thermo Scientific Pierce). Equal amounts of protein from each extract were loaded into each lane of a gel and separated by SDS-PAGE. The proteins were transferred onto PVDF membranes using standard procedures. The membranes were then blocked with $5 \%$ non-fat dry milk in TBST (TBS with $0.1 \%$ Tween 20, $\mathrm{pH} 7.6$ ) for 1 hour at room temperature (RT) and probed overnight using appropriate primary antibodies diluted in TBST (1:1000 for the anti-myc antibody and 1:1000 for the anti-Tmem10 antibody) at $4{ }^{\circ} \mathrm{C}$. After 3 washes with TBST at RT for 10 minutes each wash, the membranes were incubated with appropriate secondary antibodies diluted in TBST (1:10,000 for both the goat anti-rabbit and goat 
anti-mouse IgG antibodies) for 1 hour at RT. The membranes were washed another 3 times with TBST at RT for 10 minutes each wash, proteins were then detected with ECL reagent (Thermo Scientific Pierce) and the membranes were exposed to film (Kodak).

\section{Immunohistochemistry}

For tissue immunohistochemistry, mice aged 2-4 weeks were prepared by cardiac perfusion with cold PBS and $4 \%$ PFA followed by overnight post-fixation in the same solution at $4^{\circ} \mathrm{C}$. Fixed brains were dehydrated in $30 \%$ sucrose prior to embedding for cryosectioning. Brain sections were blocked in PBS containing $1 \%$ BSA, $10 \%$ goat serum and $0.3 \%$ Triton for 1 hour at RT and stained with appropriate primary antibodies diluted in PBS containing 1\% BSA, 1\% goat serum and $0.3 \%$ Triton overnight at $4^{\circ} \mathrm{C}$. Secondary antibodies were diluted in PBS containing $1 \%$ goat serum and incubated with the sections for 1 hour at RT. For cell-culture immunohistochemistry, OPCs from different time points were fixed with fresh $4 \%$ paraformaldehyde in PBS for 30 minutes and permeabilized by exposure to $0.1 \%$ Triton X-100 in PBS for 5 minutes. Cells were washed 3 times with PBS, blocked with PBS containing 1\% BSA, $10 \%$ goat serum and $0.3 \%$ Triton for 1 hour at RT, and stained with appropriate primary antibodies diluted in PBS containing $1 \% \mathrm{BSA}, 1 \%$ goat serum and $0.3 \%$ Triton overnight at $4^{\circ} \mathrm{C}$. Secondary antibodies were diluted in PBS with $1 \%$ goat serum and incubated with the cells for 1 hour at RT. Sections was mounted on slides with cover slips in ProLong Gold antifade reagent with DAPI (Invitrogen). The slides were dried overnight in the dark and visualized using an Olympus IX 81 microscope.

\section{Statistical analysis}

All results of Western blotting were presented as mean \pm SEM from three independent experiments at least. Data from Western blotting were analyzed by Image J software for the gray value and western blot bands were normalized against internal reference protein $\beta$-actin or GAPDH. P values were calculated using the Student's $t$ test for normally distributed data, and the value $0.05\left(^{*}\right), 0.01\left(^{* *}\right)$ and $0.001\left(^{* * *}\right)$ was assumed as the level of significance for the statistic tests carried out. All the experiments were performed for 3-6 independent experiments or with 3-6 animals for each animal experiment.

\section{Results}

\section{Generation and validation of the Tmem I 0 an- tibody}

In this study, we generated a C-terminal Tmem10-His fusion protein for the immunization of
New Zealand white rabbits. His tag is a commonly used small protein motif that can be utilized to purify tagged proteins via immobilized metal affinity chromatography. Furthermore, this rapid process allows proteins to be purified in their native state [11].

The pET22b-Tmem10-His recombinant vector was transformed into Escherichia coli BL21 cells. Expression of the Tmem10-His recombinant protein was induced with $0.25 \mathrm{mM}$ IPTG treatment for 3 hours at $35{ }^{\circ} \mathrm{C}$. The recombinant protein was $\sim 20 \mathrm{kDa}$, as determined by Coomassie blue staining following SDS-PAGE (Fig.1A). Next, we purified the Tmem10-His fusion protein using a Ni-NTA Resin to remove unwanted proteins. The purified protein was analyzed by SDS-PAGE and used to immunize New Zealand white rabbits. The successfully purified Tmem10-His fusion protein is shown in Figure 1 (Fig.1B, lane 1, arrow).

After New Zealand rabbits were immunized three times with the recombinant Tmem10-His protein, antiserum from the immunized rabbits were made and examined for its efficiency to detect recombinant and endogenous Tmem 10 proteins. To determine the titer and specificity of the Tmem10 antibody, a recombinant myc-tagged Tmem10 protein (myc-Tmem10) was overexpressed in HEK293 cells. We found that anti-sera from one of the rabbits contained Tmem10 antibody that detected multiple bands $(17-40 \mathrm{kDa})$ specific to myc-Tmem10 (Fig.1C). The detection of these multiple Tmem10 bands suggested the presence of various posttranslational modifications of Tmem10 in these cells. This result is consistent with the notion that Tmem10 is glycosylated at multiple sites $[8,10]$. In mouse brain samples, Tmem 10 appeared as a broad band between $34 \sim 40 \mathrm{kDa}$ (Fig.1D). Mouse Tmem10 is composed of 143 amino acids and has a calculated molecular mass of $\sim 16 \mathrm{kDa}$. The observed $40 \mathrm{kDa}$ band indicated that Tmem 10 was heavily glycosylated in vivo. The subsequent studies showed that the Tmem10 antibody dilution of 1:1000 was appropriate for detecting the endogenous Tmem10 in mouse brain, while the pre-immune serum detected no bands of the corresponding size (Fig.1D). We also examined different amounts of brain samples (from $1 \mathrm{ug}$ to $30 \mathrm{ug}$ of total proteins) with our Tmem10 antibody (1:1000), and we showed the increased signals with increased protein loadings (Fig.1E). All these results suggest that our Tmem10 antibody was robust in detecting endogenous Tmem10 proteins in the brain tissues.

To assay if Tmem10 antibody reacts with known myelin proteins, we used Tmem10 antibody to blot full-length gels loaded with brain extracts from the early postnatal (P2) and 3-week old mice. The results showed that we could only detect Tmem10 (34-40 
$\mathrm{kDa}$ ) in mouse brain at P22, but not in $\mathrm{P} 2$, and there were no additional bands with similar size to other myelin proteins (such as MBP, MOG, etc) (Fig.1F). Moreover, we showed that our Tmem10 antibody readily detected endogenous Tmem 10 proteins in wild type brains, but not in the Tmem10 knockout brains (Fig.1G). These results strongly validated the specificity of the Tmem10 antibody, as well as the Tmem10 knockout mice we generated. In summary, our Tmem10 antibody was confirmed to be highly specific and can detect several distinct Tmem10 bands in transfected HEK293 cells as well as in the mouse brains, suggesting that Tmem10 exists in a variety of modified forms when expressed in different cell types.

\section{Tmem I 0 is primarily expressed in mature ol- igodendrocytes in mouse CNS}

In 2006, Dugas et al. found that the mRNA levels of Tmem10 were upregulated by a fold of 23.75 during oligodendrocytes differentiation [14]. To further investigate the expression pattern of Tmem10 in vivo, we compared the expression of Tmem10 with other myelin markers, including MOG and MBP, by Western blotting analysis. In the mouse cortex, Tmem10 was firstly detected around 2 weeks after birth, which is a critical period for oligodendrocytes differentiation and myelin formation (Fig.2A). Further, we saw an age-related increase in Tmem10 protein level in mouse brain (Fig.2A).These results suggest that Tmem10 is primarily expressed in mature and myelinating oligodendrocytes, making it a potential regulator of oligodendrocytes maturation.
A

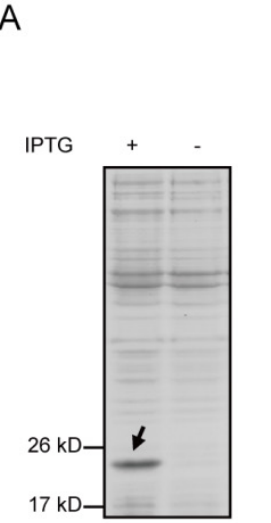

B

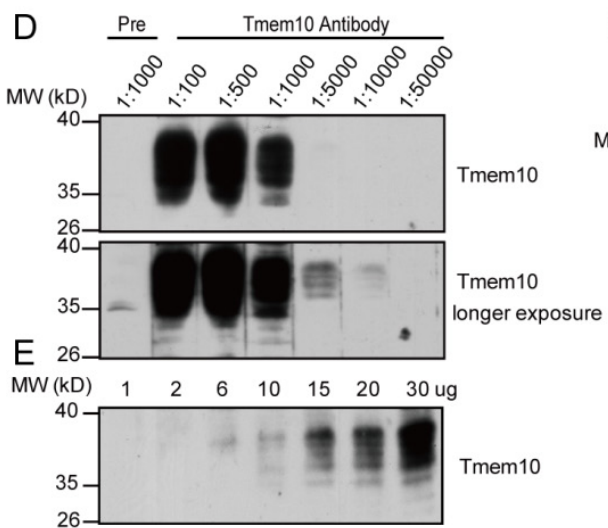

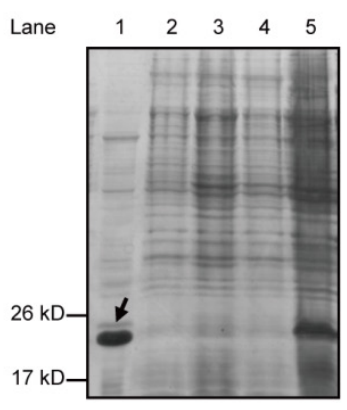

$\mathrm{F}$
C

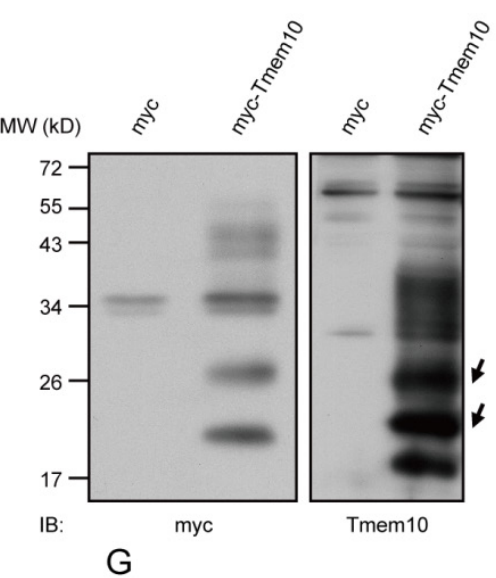

Forebrain

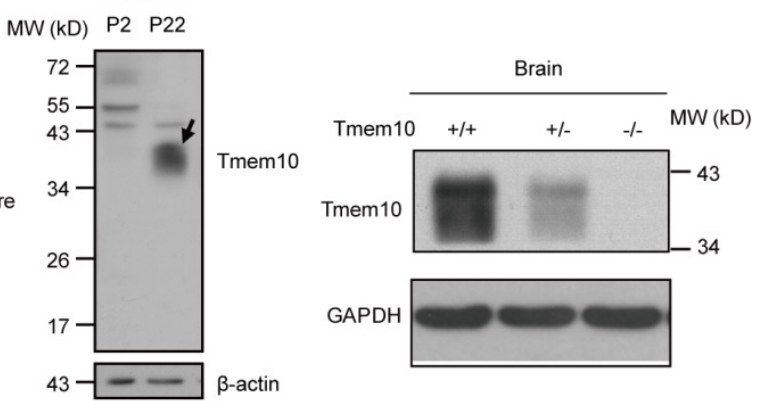

Fig. I: Generation and validation of a Tmem I0 polyclonal antibody. A: A Coomassie blue staining showing the recombinant Tmem I0-His protein (Arrow indicated). B: A Coomassie blue staining showing the purification process for the recombinant Tmem I0-His protein. Lane I-the arrow indicates the purified recombinant Tmem I0-His protein; lanes 2-4-buffers used to wash the Ni-NTA Resin at each step of the purification are shown as negative controls; Lane 5 - an unpurified sample from transformed bacteria induced to express Tmem I0-His. C: A Western blot showing that the anti-Tmem I0 antibody can specifically detect the myc-Tmem 10 recombinant protein. Note that there are multiple bands detected ranging from $17-44 \mathrm{kDa}$ in $\mathrm{HEK} 293$ cells transfected with myc-Tmem I0. D: A dilution study showing that Tmem I0 antibody worked well at I:I000 and higher concentration, while the pre-immune serum did not recognize bands like TmemI0 antibody. Pre: pre-immune rabbit serum; TmemI0 antibody with different dilution ( I: I00-I:50,000). E: A Western blot showing that Tmem I 0 antibody detected increased signals with increased protein loadings. F: A Western blot showing that the anti-Tmem 10 antibody can specifically detect Tmem 10 proteins in the mouse brain. The Tmem I 0 band is indicated with an arrow in the entire blot. E: A Western blot showing that Tmem 10 antibody does not detect any bands from the brain extracts of Tmem 10 knockout brain. 
A

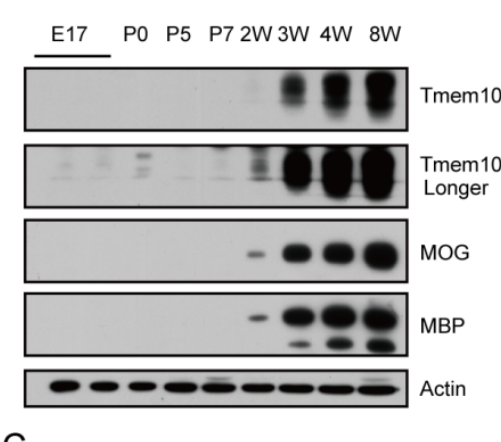

C

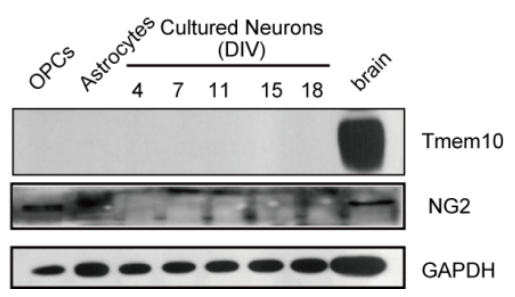

B

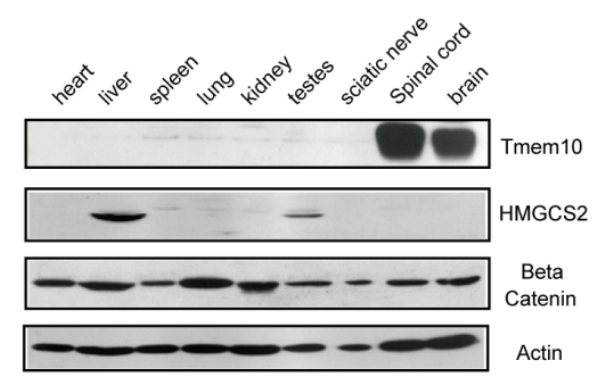

D

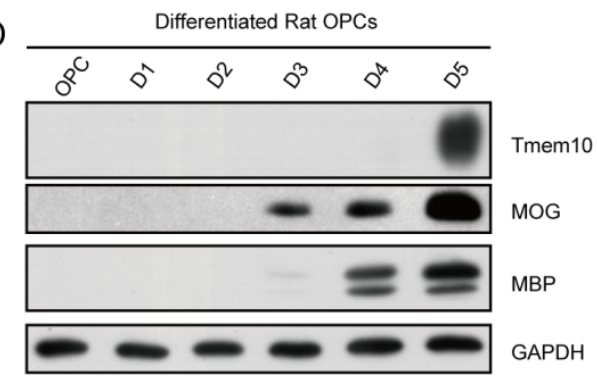

Fig. 2: Tmem 10 is primarily expressed in mature oligodendrocytes in the mouse CNS. A: Western blots showing Tmem I 0 has an expression pattern similar to that of MBP and MOG, which are first expressed around week 2 and showing an age-related increase in mouse cortex. B: Western blots showing abundant Tmem 10 expression in the mouse spinal cord and brain, but not in HMGCS2 enriched liver tissue, as detected with our Tmem 10 antibody. C: Western blots showing no expression of Tmem 10 in OPCs, astrocytes and neurons. NG2 was used as an OPC maker. D: Western blots showing Tmem 10 expression in late-stage cultured rat differentiated oligodendrocytes (D5).

Previous findings concerning the distribution of Tmem10 in various tissues have been inconsistent. Using an anti-rat Tmem10 antibody reported, Golan et al. showed that Tmem10 was expressed in the liver; however, Yoshikawa et al. were unable to detect Tmem10 mRNA in the liver $[8,10]$. In current study, we could only detect Tmem10 expression in the CNS, with no detectable expression in the liver or other tissues by our Tmem10 antibody (Fig.2B). HMGCS2 (an enzyme enriched in the liver) was used as the indicator of liver samples and beta-catenin, a widely expressed signaling molecule, as a positive control. Intriguingly, although the structure and function of myelin are very similar in the CNS and PNS (peripheral nervous system) and some myelin proteins are shared, i.e MBP, CNP, we could not detect Tmem10 expression in sciatic nerves of the peripheral nervous systems. Next, we analyzed the expression of Tmem10 in primary cultures of OPCs, astrocytes and neurons, but we were unable to detect Tmem10 in any of these cell types (Fig.2C). Especially, we could not detect Tmem10 expression in the NG2+ OPCs, indicating that Tmem10 is not expressed in OPCs. It has been reported that Tmem10 was expressed in $\mathrm{O}^{+}$ cells in cultures [9], a precursor cell stage of oligodendrocytes. Therefore, we examined the expression of Tmem10 in cultured oligodendrocytes. We found that Tmem 10 was only detectable after Day 5 in rat differentiated oligodendrocyte cultures by Western blotting (Fig.2D).
To further clarify whether Tmem10 is expressed in OPCs or not, we firstly determined the cell purity of the OPC cultures by PDGFRa/Olig2/DAPI triple staining, and found that the ratio of OPCs (PDG$\mathrm{FRa}^{+} /$Olig2 ${ }^{+}$cells) over total cells $\left(\mathrm{DAPI}^{+}\right.$cells) reached $95 \%$ (Fig.3A) by immunohistochemistry. Then we confirmed that Tmem10 was not expressed in PDGFRa ${ }^{+}$OPCs (Fig.3B), and was detectable in the cell body from Day 3, but not in the processes until Day 5 in differentiated oligodendrocytes (Fig.3C). And Tmem10 expression coincided with that of MAG, a well-known late-stage oligodendrocytes marker (Fig.3C), but its expression was later than MAG. Taken together, all these results indicate that Tmem10 is specifically expressed in late-stage oligodendrocytes.

\section{Co-expression of Tmem 10 with markers for myelinating oligodendrocytes}

To further analyze the specific expression of Tmem10, we carried out immunohistochemical assays to visualize Tmem10 expression in the brain. The anti-Tmem10 antibody was very sensitive and specific for biochemistry studies confirmed by Tmem10 KO. We examined if our Tmem10 antibody was suitable for applications in immunohistochemistry? The immunostaining results showed that our Tmem10 antibody readily detected endogenous Tmem $10^{+}$myelin fibers in wild type brains, but not in the Tmem 10 knockout brains (Fig.4A). These results validated the 
specificity of the Tmem10 antibody we generated and confirmed its use for immunohistochemistry. And we also showed that Tmem 10 could be used to label myelin in the mouse brain, as demonstrated by double staining with the well-characterized late-stage myelin marker MOG (Fig.4B). Moreover, Tmem10 was detected to be abundant in the white matter tracks of the cerebellum. The white matter tracks are regions that are enriched for mature oligodendrocytes and the Tmem10/MOG staining directly showed that Tmem10 was primarily expressed in mature oligodendrocytes, similarly to MOG. We noted that the localization of Tmem 10 was more similar to that of the late-stage myelin marker MOG than to that of the relatively early-stage myelin marker MBP. As MBP starts to express in immature oligodendrocytes, it has a much broader expression pattern than late-stage myelin markers. Taken together, our immunohistochemical results demonstrate that Tmem10 is primarily expressed in mature oligodendrocytes and can be used as a highly specific marker for myelinating oligodendrocytes.

\section{Tmem 10 expression is dramatically decreased in the brain of hypomyelination mouse model}

To further confirm that Tmem 10 could be used as a marker for impaired maturation of oligodendro-

$$
\text { A }
$$

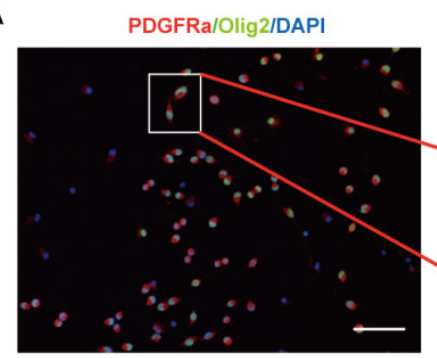

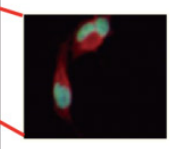

cytes and hypomyelination, we used it to examine the Tmem10 protein levels in an established hypomyelination mouse model, Rheb1-knockout mice. Rheb1 is a small highly conserved GTPase that has been shown to regulate cell growth in Drosophila and mammals, and it was reported to activate mTORC1 through direct physical interaction [15]. Our previous studies have found that Rheb1 is essential for mTORC1 signaling and myelination [12]. Therefore, Rheb1-knockout mouse serves as an excellent model for us to examine if Tmem10 is a valid marker for hypomyelination. By Western blotting, we found that Tmem 10 protein levels were reduced by $\sim 50-60 \%$ in Rheb1-knockout mice (Fig.5A and 5B). Immunohistochemical assays also showed a dramatic reduction of Tmem10 signals in multiple Rheb1-knockout tissues, including the cortex and cerebellum (Fig.5C). It is also noted that Tmem10 could label the cell body of myelinating glia and be able to quantify the number of mature oligodendrocytes (Fig.5D). All these results demonstrate that dramatic reduction of Tmem10 protein is correlated with reduction of other myelin proteins in the CNS, confirming that Tmem 10 can be used as a novel biomarker for the evaluation of hypomyelination.

B

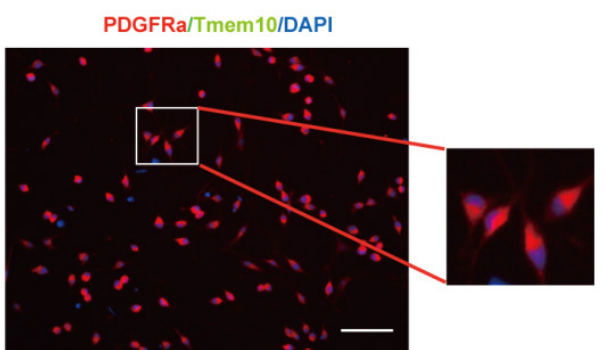

C
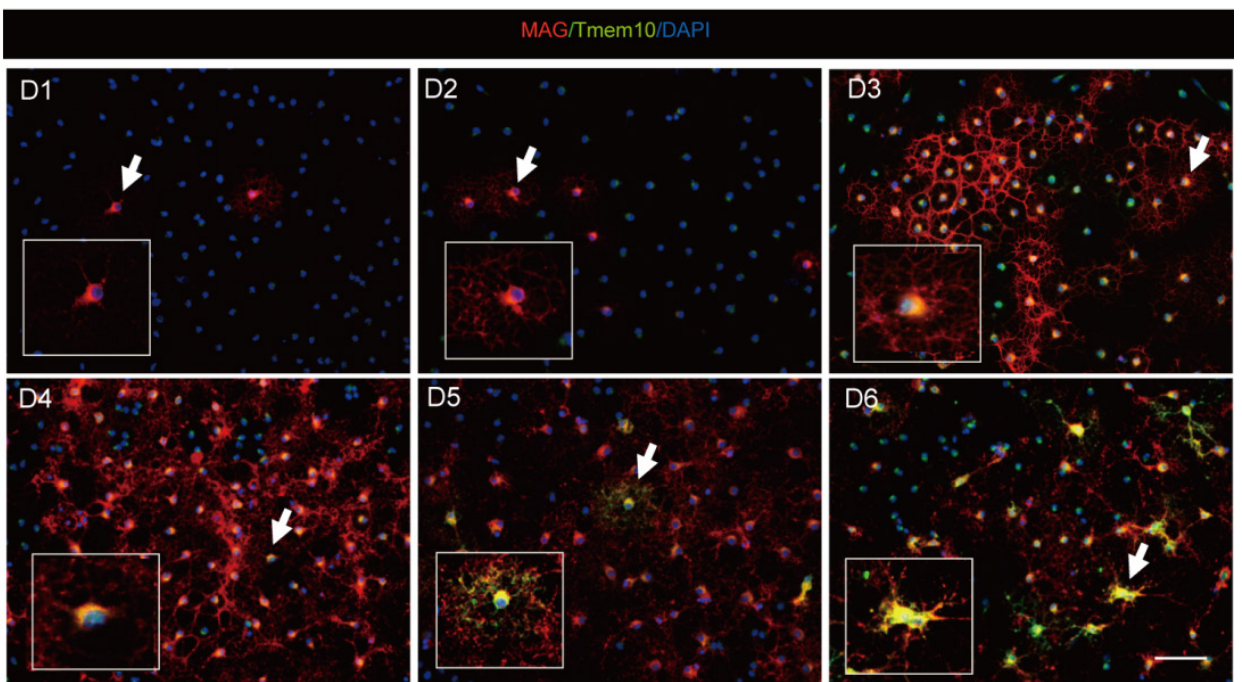

Fig.3:Tmem 10 expression coincides with that of MAG, but is not in OPCs. A: Immunostainings showing that $95 \%$ of total cultured OPCs were $\mathrm{PDGFRa}^{+} / \mathrm{Olig2}^{+}$cells. B: Immunostainings showing that Tmem 10 was not expressed in PDGFRa ${ }^{+}$cells. C: Immunostainings showing that the initial expression of Tmem 10 is later than that of MAG, a late-stage oligodendrocytes maker, and that its expression coincides with that of MAG in differentiated oligodendrocytes culture; scale bar $=50 \mu \mathrm{m}$. 


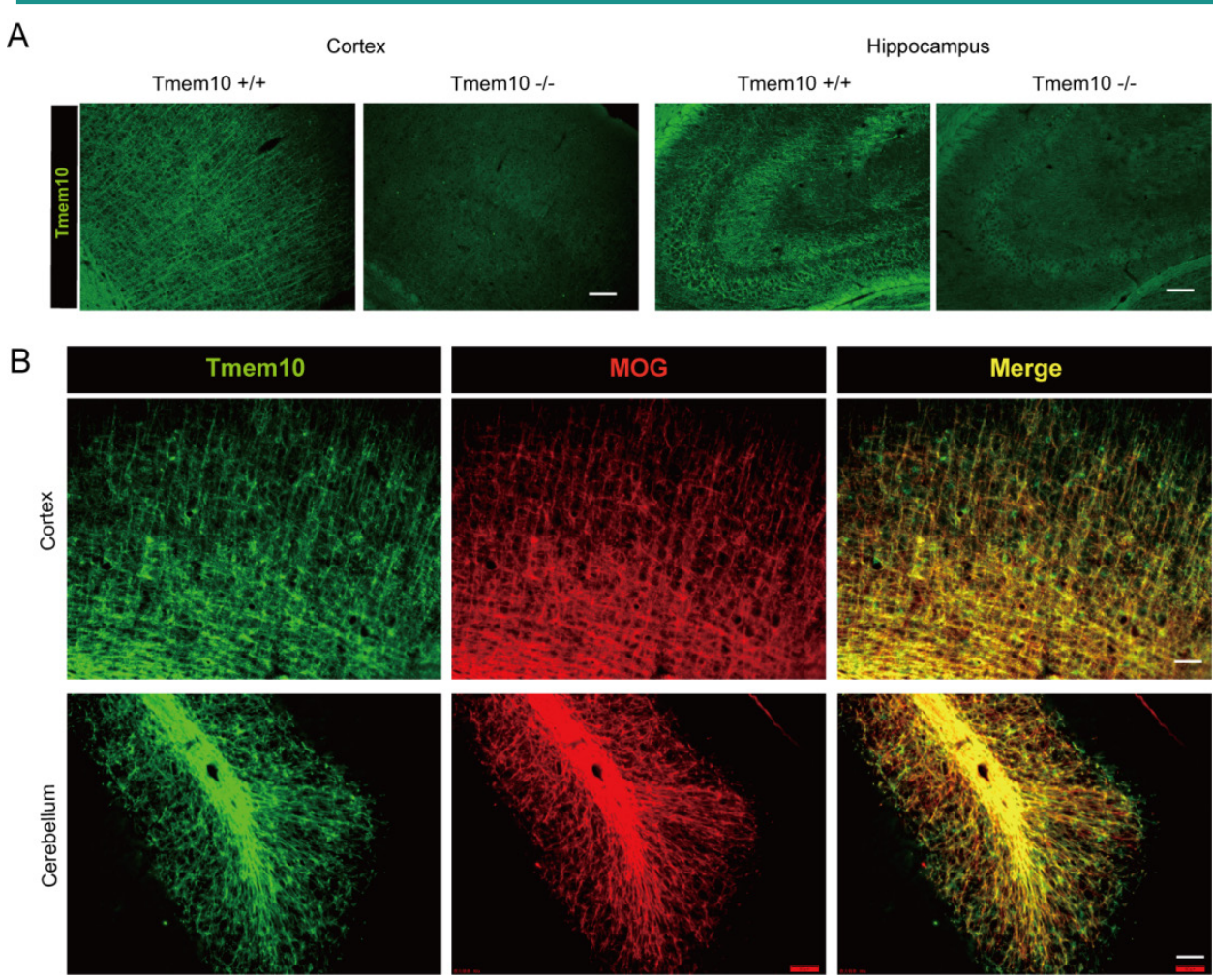

A

B

Fig. 4: Co-expression of Tmem I0 with markers for myelinating oligodendrocytes. A: Immunostainings showing that Tmem 10 antibody does not detect any Tmem I0+ myelin fibers from the Cortex and Hippocampus of Tmem 10 knockout brain; scale bar $=40$ $\mu \mathrm{m}$. B: Brain sections were dual stained for TmemlO and MOG to show the distribution of TmemlO in the cortex (CTX) and cerebellum (CB) of week 8 mouse. Tmeml0 expression was co-expressed with the late-stage myelin marker MOG; scale bar $=50 \mu \mathrm{m}$.

Fig. 5: Tmem 10 expression is dramatically decreased in the brain of hypomyelination mouse model. A: Western blots showing a $\sim 50-60 \%$ reduction in Tmem 10 protein levels in the 4-week old Rhebl-knockout mice. B: Histograms quantifying the reductions in myelin proteins in the brains of 4-week-old Rhebl-knockout mice. Data are shown as the mean \pm SEM values and are the result of three independent experiments $(* * \mathrm{p}<0.0 \mathrm{I}$ and $* * * \mathrm{p}<0.00 \mathrm{I})$. C:Immunostainings with Tmem I 0 and MBP antibodies show similar degrees of hypomyelination in the cortices of 4-week-old Rhebl knockout mice; scale bar $=100 \mu \mathrm{m}$. D:Immunostainings show cell body of myelinating oligodendrocytes identified by Tmem 10 antibody and reduced number of mature oligodendrocytes in Rhebl knockout mice; scale bar $=50 \mu \mathrm{m}$.
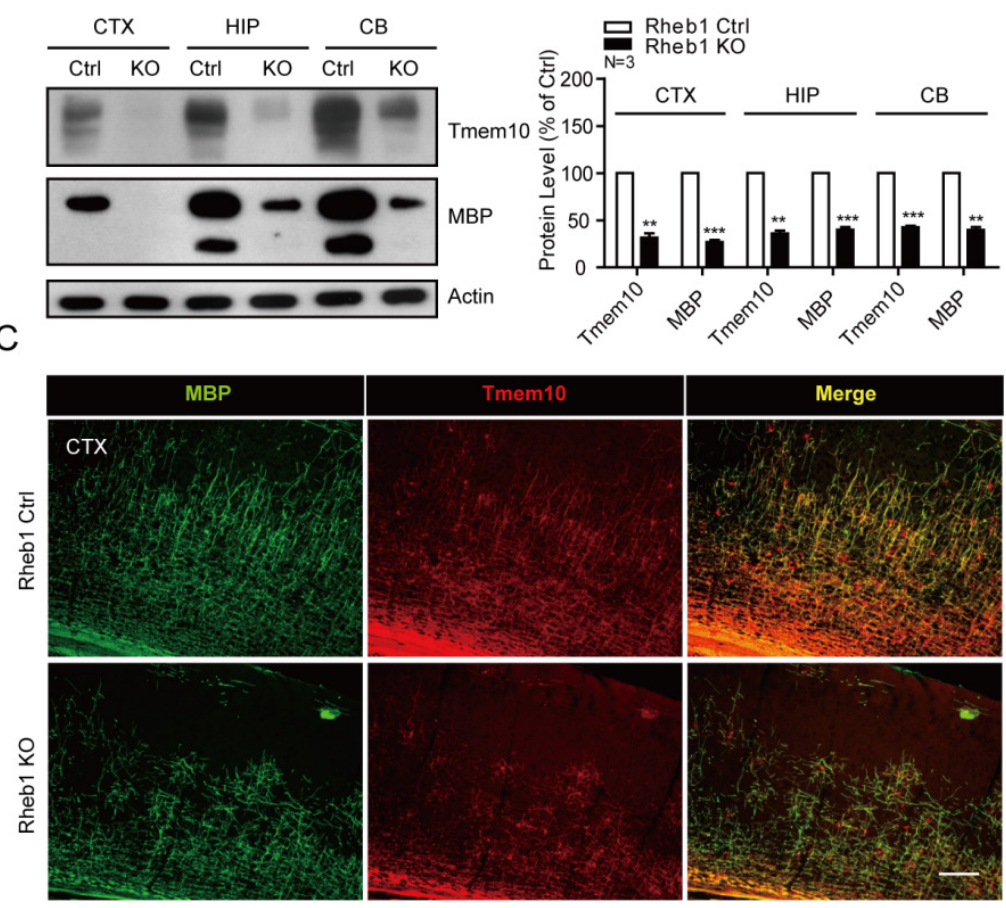

D

Rheb1 Ctrl

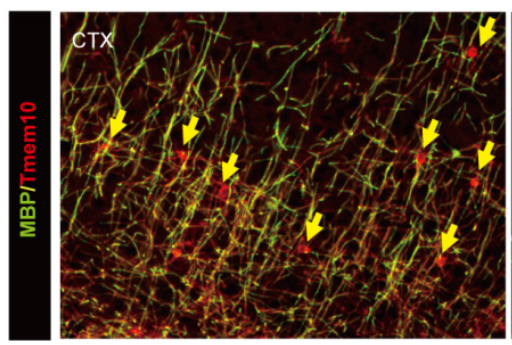

Rheb1 KO

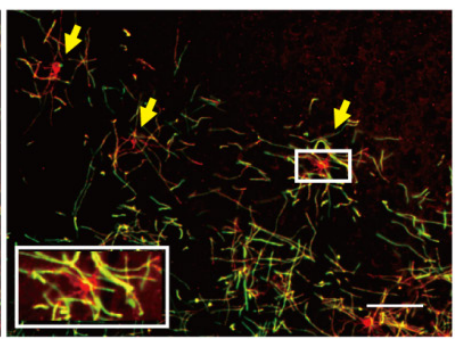




\section{Discussion}

In this study, we have described a specific Tmem10 antibody that was used to clarify that Tmem10 protein was not expressed in the early postnatal stage, but expressed at the stage where myelination is highly active. Its protein was detectable in the 2-week old postnatal mouse brain, specifically in the late stage oligodendrocytes with age-related increase. Our studies have demonstrated that Tmem10 is selectively expressed in the central nervous system. In the brain, Tmem10 co-expressed with MOG- and MBP-positive myelin fibers and was dramatically reduced in a hypomyelination mouse model. In conclusion, our study demonstrates that Tmem10 can be used as a specific marker for myelinating oligodendrocytes, which should facilitate the future studies of myelination diseases, such as multiple sclerosis.

Many neurological and psychiatric disorders, such as MS and leukodystrophy, are associated with or caused by oligodendrocytes dysfunction. However, the regulatory mechanisms that control myelination and maintain axonal integrity-the main functions of oligodendrocytes-remain largely unknown. Previous studies suggest that genes enriched in myelin-forming cells may play important roles in the CNS. For example, mice with MBP mutations showed loss of myelination[16], and MOG-deficient mice were found to lack immune tolerance[17]. Furthermore, CNP- and PLP-knockout mice show axonal degeneration $[2,4]$. In the CNS, communication between axons and ensheathing glia-oligodendrocytes occurs throughout development and adulthood, and these interactions are more complex than that in the PNS. We propose that Tmem10 may play an important role in axon-glia interactions. Future studies of the Tmem10-knockout mice would reveal its biological functions in vivo.

As a step towards elucidating these mechanisms, novel proteins that are specifically expressed in oligodendrocytes must be identified and characterized.

In this study, we focus on the novel oligodendrocytes marker gene Tmem10. The results from previous studies concerning Tmem10 are inconsistent, especially on its expression pattern. For example, Western blotting results showed that Tmem10 was expressed in the brain and liver [8], whereas RT-PCR experiments could only detect Tmem 10 mRNA in the brain [10]. Researchers using reporter gene assays in transgenic mice were also unable to fully characterize the expression pattern of Tmem10 in various tissues and cell types [18], as they found significant expression in the central nervous system, skeletal muscle, heart and kidney among the transgenic mice that showed X-gal-staining positive signals. One study showed that Tmem10 was expressed in $\mathrm{O}^{+}$cells, a precursor cell stage [9], while another report shows that it is co-localized with myelinated axons[8]. In vivo studies have been carried out to compare the expression patterns of Tmem10 and MBP, an early-stage myelination marker [14], showing that MBP is expressed earlier than Tmem10 during myelination [8, 10]. Studies involving late-stage myelin markers, such as MOG, MAG and Tmem10, are particularly useful for revealing the molecular mechanisms of myelin formation. Previous reports suggest that Tmem10 may play an important role in myelination and the maintenance of axonal integrity [8-10]; however, due to the inconclusive nature of previous studies on the expression pattern of Tmem10; it is not a widely used oligodendrocytes marker. Therefore, the highly specific anti-Tmem10 antibody we generated will help determine the true expression pattern of Tmem 10 and identify its function within the CNS. We clearly showed that Tmem10 was specifically expressed in mature oligodendrocytes in the CNS. In cultured cells, Tmem10 was undetectable in OPCs, neurons and astrocytes, but it was detectable in the processes of late-stage differentiated oligodendrocytes (Days 5-6). We showed that Tmem10 was firstly expressed at a later stage than MBP (an early-stage myelin marker) and MAG (a late-stage myelin marker).

In present study, we also showed that Tmem10 expression was dramatically decreased in a hypomyelination mouse model, namely, a Rheb1-knockout mouse for the first time. In a previous paper, we characterized widespread hypomyelination during postnatal stages in a Rheb1-knockout mouse [12]. Now we extended to show that Tmem10 expression was reduced by $50-60 \%$ in Rheb1-knockout mouse. Thus, we propose that Tmem10 can be used as a myelin marker to evaluate the extent of hypomyelination and myelination-related human diseases, such as MS.

\section{Acknowledgments}

We thank Yiyuan Cui and Xiaoqiang Xia for technical assistance. The authors of this manuscript have no financial interest related to this work. This study was funded by a grant from National 973 Basic Research Program of China (2009CB941400) and National Natural Science Foundation of China (30800311).

\section{Competing Interests}

The authors have declared that no competing interest exists.

\section{References}

1. Nave KA. Myelination and support of axonal integrity by glia. Nature. 2010; 468: 244-52. doi:10.1038/nature09614. 
2. Lappe-Siefke C, Goebbels S, Gravel M, Nicksch E, Lee J, Braun PE, et al. Disruption of Cnp1 uncouples oligodendroglial functions in axonal support and myelination. Nat Genet. 2003; 33: 366-74. doi:10.1038/ng1095.

3. Li C, Tropak MB, Gerlai R, Clapoff S, Abramow-Newerly W, Trapp B, et al. Myelination in the absence of myelin-associated glycoprotein. Nature. 1994; 369: 747-50. doi:10.1038/369747a0.

4. Griffiths I, Klugmann M, Anderson T, Yool D, Thomson C, Schwab MH, et al. Axonal swellings and degeneration in mice lacking the major proteolipid of myelin. Science. 1998; 280: 1610-3.

5. Barnett $\mathrm{MH}$, Sutton I. The pathology of multiple sclerosis: a paradigm shift. Curr Opin Neurol. 2006; 19: 242-7.

6. Gold R, Linington C, Lassmann H. Understanding pathogenesis and therapy of multiple sclerosis via animal models: 70 years of merits and culprits in experimental autoimmune encephalomyelitis research. Brain. 2006; 129: 1953-71. doi:10.1093/brain/awl075.

7. Orchard PJ, Wagner JE. Leukodystrophy and gene therapy with a dimmer switch. N Engl J Med. 2011; 364: 572-3. doi:10.1056/NEJMcibr1013082.

8. Golan N, Adamsky K, Kartvelishvily E, Brockschnieder D, Mobius W, Spiegel I, et al. Identification of Tmem10/Opalin as an oligodendrocyte enriched gene using expression profiling combined with genetic cell ablation. Glia. 2008; 56: 1176-86. doi:10.1002/glia.20688.

9. Kippert A, Trajkovic K, Fitzner D, Opitz L, Simons M. Identification of Tmem10/Opalin as a novel marker for oligodendrocytes using gene expression profiling. BMC Neurosci. 2008; 9: 40. doi:10.1186/1471-2202-9-40.

10. Yoshikawa F, Sato Y, Tohyama K, Akagi T, Hashikawa T, Nagakura-Takagi Y, et al. Opalin, a transmembrane sialylglycoprotein located in the central nervous system myelin paranodal loop membrane. J Biol Chem. 2008; 283: 20830-40. doi:10.1074/jbc.M801314200.

11. Gibert S, Bakalara N, Santarelli X. Three-step chromatographic purification procedure for the production of a his-tag recombinant kinesin overexpressed in E. coli. J Chromatogr B Biomed Sci Appl. 2000; 737: 143-50.

12. Zou J, Zhou L, Du XX, Ji Y, Xu J, Tian J, et al. Rheb1 is required for mTORC1 and myelination in postnatal brain development. Dev Cell. 2011; 20: 97-108. doi:10.1016/j.devcel.2010.11.020.

13. Yang Z, Watanabe M, Nishiyama A. Optimization of oligodendrocyte progenitor cell culture method for enhanced survival. J Neurosci Methods. 2005; 149: 50-6. doi:10.1016/j.jneumeth.2005.05.003.

14. Dugas JC, Tai YC, Speed TP, Ngai J, Barres BA. Functional genomic analysis of oligodendrocyte differentiation. J Neurosci. 2006; 26: 10967-83. doi:10.1523/JNEUROSCI.2572-06.2006.

15. Long $X$, Lin $Y$, Ortiz-Vega $S$, Yonezawa $K$, Avruch J. Rheb binds and regulates the mTOR kinase. Curr Biol. 2005; 15: 702-13. doi:10.1016/j.cub.2005.02.053.

16. Chernoff GF. Shiverer: an autosomal recessive mutant mouse with myelin deficiency. J Hered. 1981; 72: 128.

17. Delarasse C, Daubas P, Mars LT, Vizler C, Litzenburger T, Iglesias A, et al Myelin/oligodendrocyte glycoprotein-deficient (MOG-deficient) mice reveal lack of immune tolerance to MOG in wild-type mice. J Clin Invest. 2003; 112: 544-53. doi:10.1172/JCI15861.

18. Aruga J, Yoshikawa F, Nozaki Y, Sakaki Y, Toyoda A, Furuichi T. An oligodendrocyte enhancer in a phylogenetically conserved intron region of the mammalian myelin gene Opalin. Journal of neurochemistry. 2007; 102: 1533-47. doi:10.1111/j.1471-4159.2007.04583.x. 